\title{
Negative Differential Conductivity in InAs/AlSb Superlattices
}

\author{
M.S. KAgAn ${ }^{a}$, I.V. Altukhov ${ }^{a}$, A.N. BArAnov ${ }^{b}$, N.D. Il'Ynskaya ${ }^{c}$, S.K. PaprotskiY ${ }^{a}$, \\ V.P. Sinis ${ }^{a}$, R. TEISSIER ${ }^{b}$ AND A.A. UsIKOVA ${ }^{c}$ \\ ${ }^{a}$ V.A. Kotel'nikov Institute of Radio Engineering and Electronics, Russian Ac. Sci., Moscow, Russia \\ ${ }^{b}$ IES, Université Montpellier 2, CNRS, Montpellier, France \\ ${ }^{c}$ A.F. Ioffe Physico-Technical Institute, Russian Ac. Sci., St. Petersburg, Russia
}

\begin{abstract}
The negative differential conductivity and electric instabilities are found to appear in type-II InAs/AlSb superlattices. The origin of the nonlinear effects is discussed.
\end{abstract}

PACS: 72.20.Ht, 73.50.Fq, 73.63.-b

\section{Introduction}

In recent years, much attention has been focused on studies of InAs-based type-II heterostructures due to a high conduction band offset and a small electron effective mass of $0.023 m_{0}$ in InAs. The studies have been performed mainly on InAs / AlSb structures aimed for mid-IR (see, e.g., [1] and references within) and $\mathrm{THz}$ [2] photodetectors, quantum cascade lasers operating in the spectral range of 2.3 to $5 \mu \mathrm{m}$ (e.g., [3]) and, recently, fast hot-electron transistors [4]. Transport properties of superlattices (mainly GaAs-based SLs) have been investigated in detail in connection with expected Bloch wave amplification. It was found that negative differential conductivity (NDC) causes the formation of electric domains, which makes it difficult to observe the Bloch oscillations. InAs-based SLs are also very attractive for studies of these nonlinear phenomena. In this report we present studies of vertical transport in InAs/AlSb SLs.

\section{Experimental}

The InAs/AlSb SL samples have been grown on InAs substrate by molecular beam epitaxy at Montpellier University. The SL consists of 85 periods of $4 \mathrm{~nm}$ $\mathrm{InAs} / 2 \mathrm{~nm}$ AlSb between $n^{+}$top contact layer of $\operatorname{InAs}\langle\mathrm{Si}\rangle$ $\left(n=1 \times 10^{19} \mathrm{~cm}^{-3}\right)$ and $n^{+} \operatorname{InAs}\langle\mathrm{Si}\rangle$ substrate $(n=$ $\left.2 \times 10^{18} \mathrm{~cm}^{-3}\right)$. The Si doping level of SLs was $(1 \div$ 2) $\times 10^{17} \mathrm{~cm}^{-3}$. The structures have been processed by means of standard wet etching techniques as well as classical lithography. The last step was a metal (gold) deposition. Different mesa structures have been designed, with areas from $10^{-3}$ to $10^{-2} \mathrm{~cm}^{2}$. Voltage pulses of 0.2 to $10 \mu$ s duration were applied to the sample and the load resistance connected in series. The time dependences of voltages on the sample and load resistance were recorded. Microwave radiation from the samples was registered by means of either mw detector or thermistor heads.

\section{Results and discussion}

Figure 1 shows the current-voltage characteristics of a SL sample measured at the end of sufficiently long

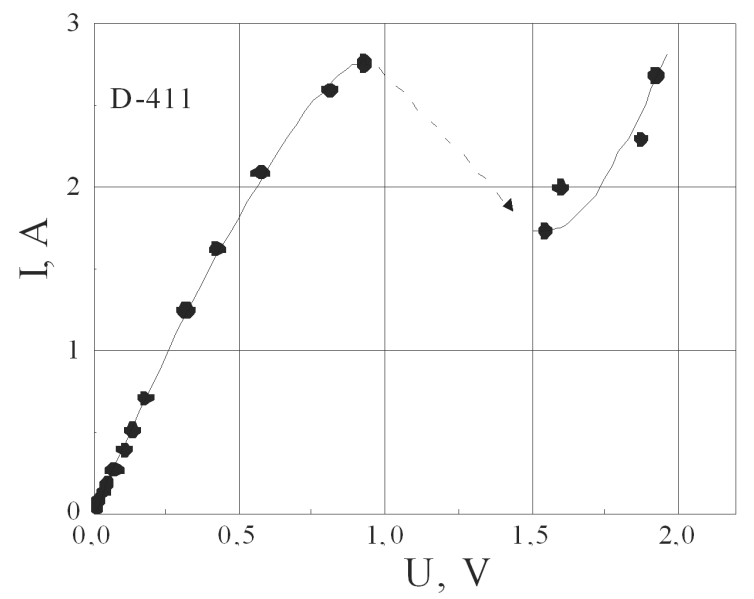

Fig. 1. $I-V$ characteristics of InAs/AlSb SL at $77 \mathrm{~K}$.

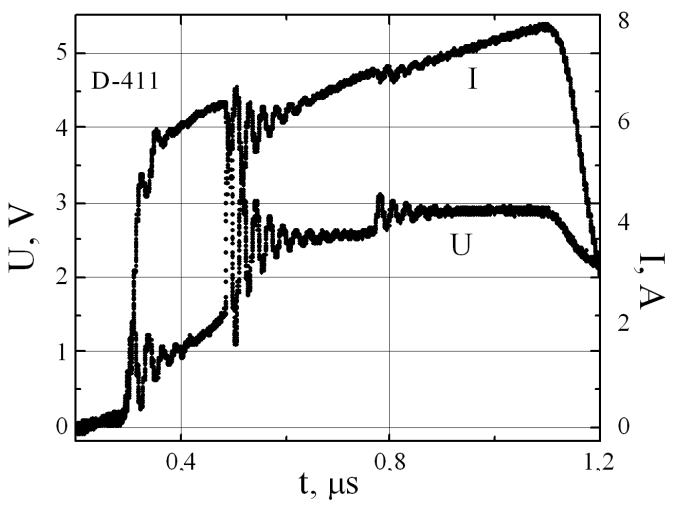

Fig. 2. Current and voltage pulse shapes. $T=77 \mathrm{~K}$.

(several $\mu \mathrm{s}$ ) voltage pulse when both voltage and current saturated. The region with NDC is clearly seen. Of course, the NDC is only imaginary because the domain formation should destroy the field uniformity. The arrow in Fig. 1 shows the current jump resulting from matched load resistance. The NDC is supposed to be due to the well-known Esaki-Tsu mechanism for mini- 


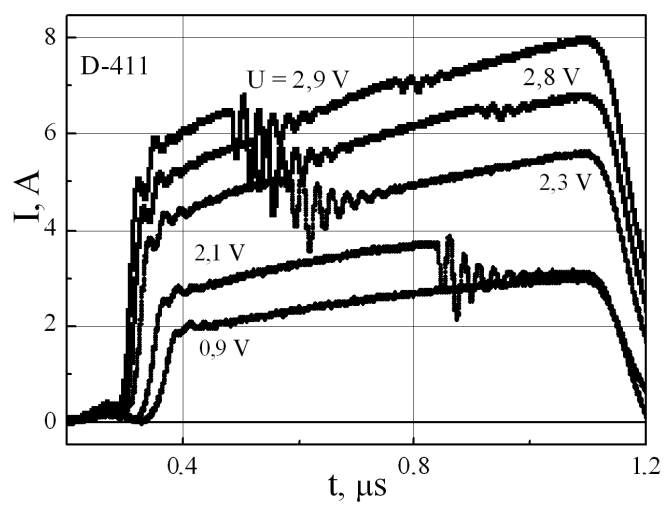

Fig. 3. Current pulses at different applied voltages.

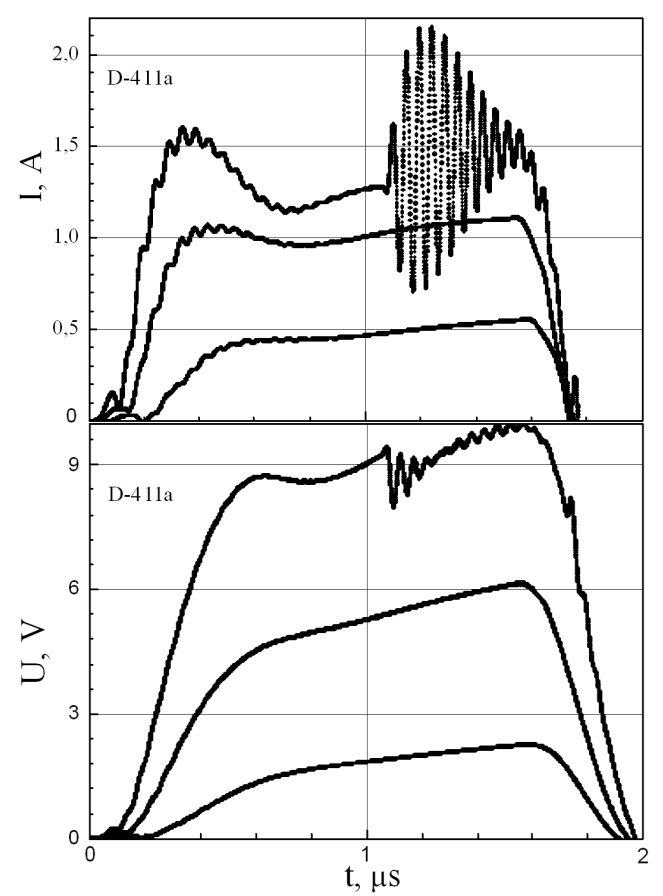

Fig. 4. Current and voltage pulses at room temperature. Successive curves relate to increasing voltages.

band transport in SLs. Kinetics of the sample voltage and current is presented in Fig. 2 for maximal applied voltage. It is rather complicated. The damped oscillations arisen at the time moments 0.48 and $0.77 \mu$ s are due to parasitic resonant circuits and indicate the appearance of NDC. Time averaging over the oscillations shows the jumps in the sample resistance. Two such kinks are seen in Fig. 2. They are likely connected with field domain formation.

The current evolution at different voltages is shown in Fig. 3. The time positions of the current kinks move to the start simply due to increasing voltage with time; it is due to the Joule heating the sample during the pulse. The jumps (and the following parasitic oscillations) appear at the same instantaneous voltage. The kinetics of current and voltage at room temperature is presented in Fig. 4; it is of the same character.

Microwave radiation of 30 to $40 \mathrm{GHz}$ for different samples was registered just at voltages of the current kinks. The frequencies correspond approximately to transit-time domain oscillations.

In summary, the studies of vertical transport in type-II InAs/AlSb superlattices reveal the appearance of NDC and field domains in this system.

\section{Acknowledgments}

The work is supported by RFBR grants 07-02-00857, 08-02-01464, 08-02-91323 and RAS programs "Basic research in nanotechnology and nanomaterials" and "Modern problems of radiophysics".

\section{References}

[1] J. Hoffmann, T. Lehnert, D. Hoffmann, H. Fouckhardt, Semicond. Sci. Technol. 24, 065008 (2009); J. Guo, Z. Peng, W. Sun, Y. Xu, Z. Zhou, Z. Niu, Infrared Phys. Technol. 52, 124 (2009); J.B. Rodriguez, E. Plis, G. Bishop, Y.D. Sharma, H.S. Kim, L.R. Dawson, S. Krishna, Appl. Phys. Lett. 91, 043514 (2007).

[2] L.L. Li, W. Xu, Z. Zeng, A.R. Wright, C. Zhang, J. Zhang, Y.L. Shi, T.C. Lu, Microelectron. J. 40, 812 (2009).

[3] A.N. Baranov, N. Bertru, Y. Cuminal, G. Boissier, C. Alibert, A. Joullié, Appl. Phys. Lett. 71, 735 (1997); A.A. Popov, V.V. Sherstnev, A.N. Baranov, C. Alibert, Y.P. Yakovlev, Electron. Lett. 34, 1398 (1998); J. Devenson, R. Teissier, O. Cathabard, A.N. Baranov, Appl. Phys. Lett. 90, 111118 (2007).

[4] T. Daoud, G. Boissier, J. Devenson, A.N. Baranov, R. Teissier, J. Phys., Conf. Series 193, 012014 (2009). 